\title{
Arbitrary numbers counter fair decisions: trails of markedness in card distribution
}

\author{
Philipp A. Schroeder ${ }^{1 *}$ and Roland Pfister ${ }^{2}$ \\ ' Department of Psychiatry and Psychotherapy, Neurophysiology and Interventional Neuropsychiatry, University of Tübingen, Tübingen, Germany \\ ${ }^{2}$ Department of Psychology III, University of Würzburg, Würzburg, Germany
}

\author{
Edited by: \\ Hans-Christoph Nuerk, University of \\ Tübingen, Germany \\ Ann Dowker, University of Oxford, \\ UK \\ Reviewed by: \\ Stefan Huber, Knowledge Media \\ Research Center, Germany \\ Tobias Loetscher, University of \\ South Australia, Australia \\ *Correspondence: \\ Philipp A. Schroeder, Department of \\ Psychiatry and Psychotherapy, \\ Neurophysiology and Interventional \\ Neuropsychiatry, University of \\ Tübingen, Calwerstrasse 14, \\ D-72076 Tübingen, Germany \\ e-mail:philipp.schroeder@ \\ uni-tuebingen.de
}

Converging evidence from controlled experiments suggests that the mere processing of a number and its attributes such as value or parity might affect free choice decisions between different actions. For example the spatial numerical associations of response codes (SNARC) effect indicates the magnitude of a digit to be associated with a spatial representation and might therefore affect spatial response choices (i.e., decisions between a "left" and a "right" option). At the same time, other (linguistic) features of a number such as parity are embedded into space and might likewise prime left or right responses through feature words [odd or even, respectively; markedness association of response codes (MARC) effect]. In this experiment we aimed at documenting such influences in a natural setting. We therefore assessed number-space and parity-space association effects by exposing participants to a fair distribution task in a card playing scenario. Participants drew cards, read out loud their number values, and announced their response choice, i.e., dealing it to a left vs. right player, indicated by Playmobil characters. Not only did participants prefer to deal more cards to the right player, the card's digits also affected response choices and led to a slightly but systematically unfair distribution, supported by a regular SNARC effect and counteracted by a reversed MARC effect. The experiment demonstrates the impact of SNARC-and MARC-like biases in free choice behavior through verbal and visual numerical information processing even in a setting with high external validity.

Keywords: embodied cognition, numerical cognition, SNARC effect, MARC effect, and justice for all, linguistic markedness, free choice

\section{INTRODUCTION}

Like nothing else, numbers are regarded as pure and objective. They are the cornerstone of scientific progress in terms of measurements and statistics and they similarly shape global business in various ways-from defining monthly salaries to describing trends at the stock market. But does this objectivity survive when numbers come in contact with human agents? In fact, there seems to be good reason for a positive answer to this question. Numbers obviously allow for rule-based decisions between competing options, and a decision that is based on numbers is readily accepted as fair and impersonal (Porter, 1996). At the same time, however, research on human decision making has documented that numbers can systematically bias an agent's choice behavior via anchoring and adjustment heuristics (Mussweiler and Englich, 2003; Furnham and Boo, 2011). For instance, when asked to estimate the value of a property, laymen and professionals alike rated the price of a real estate higher when they were told a higher listed price before (Northcraft and Neale, 1987). This anchoring bias was found in numerous contexts and research in this domain has shown that heuristic decisions might even integrate nominally irrelevant anchors like telephone and social insurance numbers (Tversky and Kahneman, 1974).
Such anchoring effects are of course driven by memory processes rather than by the numbers themselves. Still, recent research on numerical and embodied cognition suggests that the mere presence of a number alone might be sufficient to invoke biases in thoughts and actions (Barsalou, 1999; Fischer, 2006, 2012). These biases built on well-documented associations between numerical magnitude and spatial locations that indicate smaller numbers to be associated with left locations and larger numbers to be associated with right locations [spatial numerical associations of response codes (SNARC) effect; Dehaene et al., 1993; Wood et al., 2008]. Most importantly for the present study, such spatial-numerical associations also affect response choices (Tschentscher et al., 2012; Shaki and Fischer, 2014). That is, when being confronted with smaller numbers, participants showed a preference for choosing a left vs. a right response key (Daar and Pratt, 2008) and, similarly, such small numbers involuntarily prompted left-oriented gaze directions (Ruiz Fernández et al., 2011) and small numbers were produced more likely while turning or gazing to the left (Loetscher et al., 2008, 2010). These automatic biases document that the mere presence of a number is sufficient to bias choices and behavior. Sensory and motor biases induced by the SNARC effect can be considered of high 


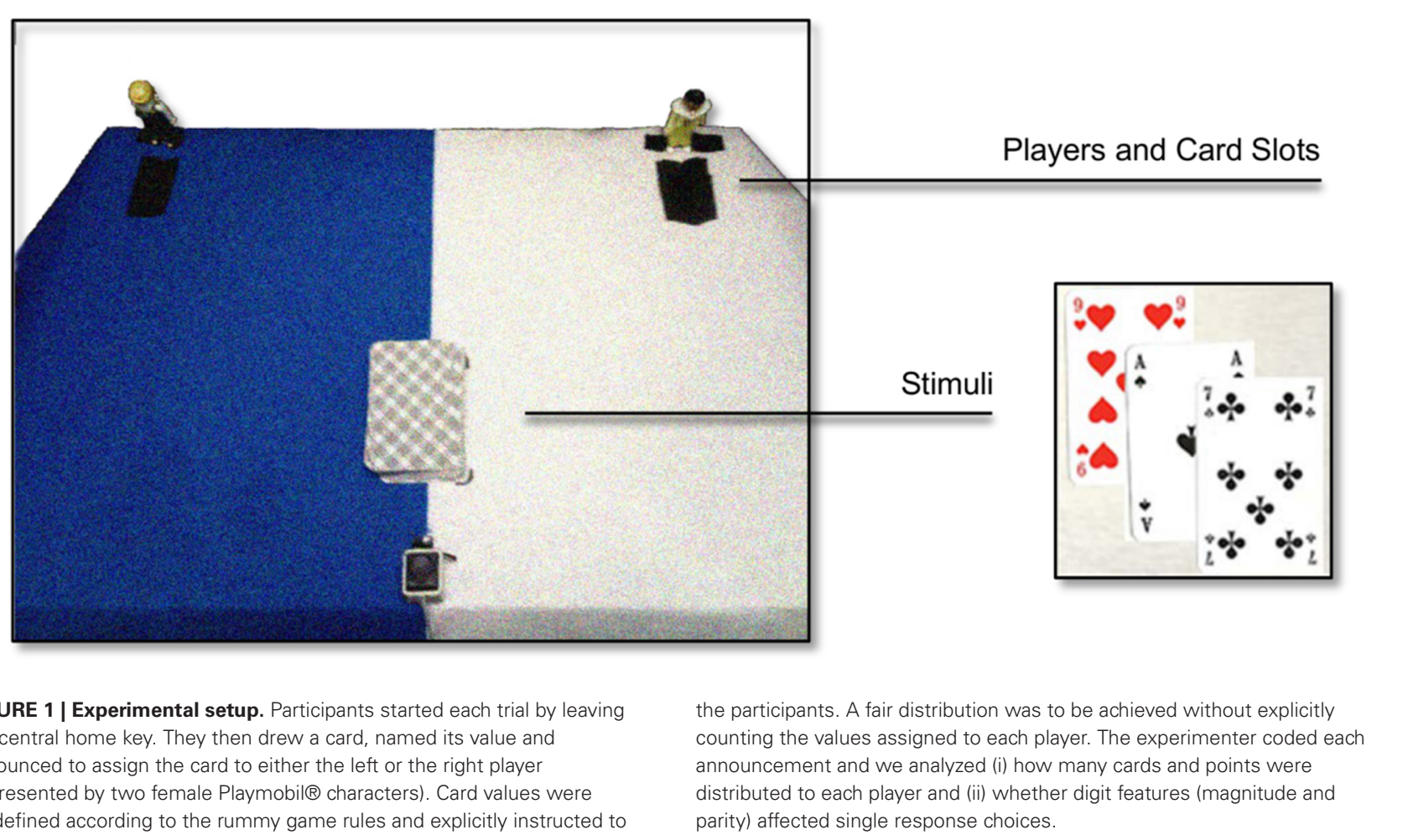

diagnostic merit for the understanding of grounded, embodied, and situated cognition (Fischer, 2012). Findings pertinent to this point range from culture-dependent finger counting habits that influence magnitude representations (Domahs et al., 2010) to bodily postures (Eerland et al., 2011) or even "unusual bodies" (Keehner and Fischer, 2012) that introduce peculiarities in spatial tasks. Together, these studies indicate that numerical associations reliably alter spatial response choices in deliberately employed highly controlled settings where the agent does not pursue any other goals except for deciding spontaneously for a spatially coded response.

As a first aim, the present study investigated whether the described bias would also occur in a more externally valid setting such as in situations where the agent aims at fairly and objectively distributing value among other people. We operationalized this situation in terms of a card distribution task in which participants were asked to deal cards of a given value to a player to the left or to the right and additionally announce their value-space choice (Figure 1). If spatial-numerical biases do indeed generalize to this situation, participants should deal more cards with higher values to the right player than to the left player.

Of course, these biases do not work in an all or none fashion, but gradually. That is, even though participants prefer choices that are congruent to a number's spatial association (e.g., a left response to a small number), they also tend to show a fair amount of incongruent choices (e.g., a right response to a small number; Daar and Pratt, 2008). In the natural card playing setting of this study, however, both spatial-numerical associations and markedness of parity and space [markedness association of response codes (MARC) effect; Nuerk et al., 2004] might affect choice probabilities for each single card, summing up to an overall biased and therefore unfair bias in value distribution. As both, high and even numbers (such as the target card value " 8 " or " 10 ") are usually associated with right responses and with more points in the rummy card setting at hand, our main hypothesis was that participants would be biased to deal overall more points to the right than to the left player.

\section{MATERIALS AND METHODS PARTICIPANTS AND APPARATUS}

Twenty-five participants ( 19 females, mean age $=24.3$, range: 18-52 years, 3 left-handed) ${ }^{1}$ were invited to participate in a 15-min experimental session. They were seated in front of the apparatus displayed in Figure 1. This apparatus mainly consisted of a $60 \times 40 \mathrm{~cm}$ cardboard box, the surface of which was covered with blue and white paper. Two Playmobil ${ }^{\circledR}$ characters represented the players and were positioned at the rear edge of the card box surface with an inter-player distance of $50 \mathrm{~cm}$. The players were matched for various attributes such as size, age, beauty, and orientation toward the participant. A slot in front of each player allowed the participants to insert a card in a box beneath the surface of the apparatus, restricting visual feedback of the current distribution. A central key was positioned at the front edge to allow for a standardized trial procedure, and the

\footnotetext{
${ }^{1}$ As pointed out by a reviewer, individual variations of age, handedness or sex might play a role in marked decisions about numbers (see the discussion for an elaboration). However, also following the reviewer's suggestion, fitting a model on right-handed female participants aged 30 or less did not substantially alter the results and only marginally improved the model fit.
} 
card deck was placed $10 \mathrm{~cm}$ from the key onto a predefined mark. One participant decided to distribute cards by color and thereby achieved a totally fair distribution; this participants' data was excluded from the analysis and we refer to the remaining $N=24$ participants in the following. The study was conducted in accordance with the Declaration of Helsinki and the guidelines of the ethics committee at the University of Würzburg.

\section{PROCEDURE}

The basic task of the participants was to draw a card and deal it to either the left or the right player. Each participant received three random training cards, then a complete 52 Anglo-American style rummy card pack. We ensured that the card icons were printed in all four corners of each card to avoid systematic influences originating from the specific stimulus set (Figure 1). Card values were defined following standard rummy game rules, that is: number cards (2-10) counted their printed value (i.e., two points for a " 2 ," three points for a " 3 ," and so on), royal cards (jack, queen, and king) counted 10 points, and aces counted 11 points. The deck was professionally shuffled prior to the experiment. During the instructions, we emphasized that participants should aim for a fair distribution of values across players by intuition and without using any explicit strategies (such as counting points across the experiment).

To start a trial, participants pressed and released the start button. They then drew the top card from the deck, read out loud the card's face (e.g., "Ace of Spades"), its value ("11"), and announced the side they wanted to distribute it to (always in this order). They then inserted the card into the right or left card slot. The experimenter registered the information and also coded invalid trials (i.e., illegal use of the left hand, reading out the wrong number or value, or naming the card's attributes and the corresponding choice in the wrong order; $4.4 \%$ trials in total).

\section{DATA TREATMENT}

For the main analysis, both the number of cards and the resulting scores for each player and participant were computed. Note that although the two measures are confounded, they still allow for distinct evaluation of choice preference and influences of the SNARC or the MARC effect: Even without an overall preference of one player in terms of the number of cards, a difference in scores can arise from a SNARC-like distribution of high-value cards to the right player and low-value cards to the left player. Both measures were controlled for homogeneity and normal distribution and subjected to one-tailed paired $t$-tests to assess our main hypothesis of a preference for the right player.

In a second, exploratory analysis, we aimed at dismantling underlying SNARC and MARC influences to the free, binary choice at a trial-wise level. Therefore, we used generalized mixedeffects models to predict the likelihood of a left response from the two first-level fixed factors parity and magnitude.

\section{RESULTS}

\section{SCORES AND NUMBER OF CARDS}

Mean scores and number of cards for each player are depicted in Figure 2. Tests for normal distribution (Kolmogorov Smirnov: $p s>0.23$ ) and homogeneity of the sample were conducted prior to the analysis and showed the data to be suitable for analyses via parametric tests.

Whereas $188(\mathrm{SE}=3.33)$ points on average were assigned to the right player, only $172(\mathrm{SE}=3.76)$ points were assigned to the left player, and this difference in scores was significant, $t(23)=2.52, p=0.010, d=0.53$ (Figure 2A). A similar effect emerged for the number of cards dealt to the left and right player, respectively, $t(23)=1.92, p=0.034, d=0.40$ (Figure $2 \mathrm{~B}$ ), as participants assigned about two cards more $\left(\overline{d_{N}}=1.71, \mathrm{SE}=0.48\right)$ to the right player. The effects on points and card numbers were

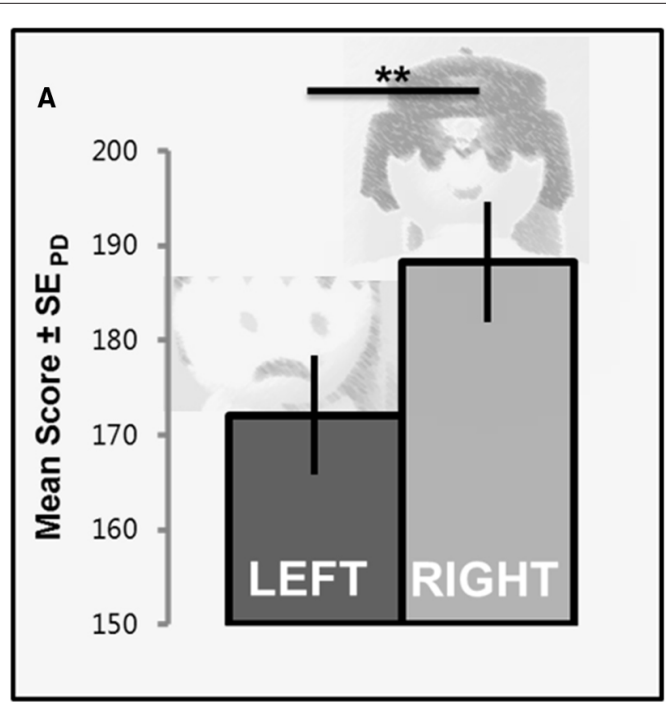

FIGURE 2 | Mean scores and standard errors of paired difference (cf. Pfister and Janczyk, 2013) in the card distribution task. Participants overall preferred the right player which resulted in a significant difference in scores

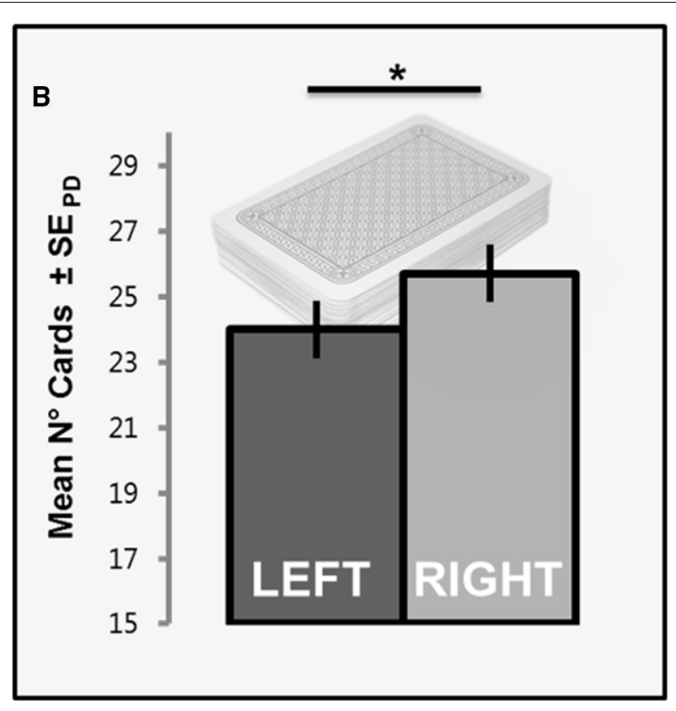

(A), and a similar effect in overall card numbers (B). Note that both Playmobil players acted earnest without any particular facial expression during the experiment, unlike the displayed emotions in panel (A). ${ }^{*} p<0.05,{ }^{*} p<0.01$ 
Table 1 | Probabilities of left response choices as a function of target card value.

\begin{tabular}{|c|c|c|c|c|c|c|c|c|c|c|}
\hline \multirow[b]{2}{*}{ Target card value } & \multicolumn{8}{|c|}{ Single-digit cards } & \multicolumn{2}{|c|}{ Royal cards } \\
\hline & 2 & 3 & 4 & 5 & 6 & 7 & 8 & 9 & 10 & 11 \\
\hline$P$ (left) [\%] & 57.3 & 50.4 & 48.3 & 42.0 & 52.8 & 40.6 & 48.5 & 42.4 & 49.4 & 47.2 \\
\hline
\end{tabular}

correlated significantly across participants, $r=0.85, p<0.001$, indicating that the difference in cards accounted for about $71 \%$ of the effect on distributed points.

\section{EXPLORATORY ANALYSIS: SNARC AND MARC EFFECTS}

More fine-grained analyses targeted the outcome of individual decisions rather than the overall number of points or cards dealt by each participant (Table $\mathbf{1}$ ). More precisely we aimed at analyzing the impact of magnitude and parity on the outcome of a decision (i.e., the likelihood for a card to be dealt to the left or to the right). To this end, we employed generalized linear mixed-effects models to model the binary outcome of the choice. Magnitude and parity were entered as fixed factors into the model (first level predictors), which further included individual subjects as random effects on the second level. The model was fitted in $\mathrm{R}$ by using the glmer function of the lme4 package (Bates et al., 2014; binomial family and logit link function). We further restricted the analysis to single-digit values (2-9) due to the actual different pictorial presentation of royal card values and possibly different representational format of values that would imply a two-digit numerical notation (Nuerk and Willmes, 2005; Nuerk et al., 2011).

In a first step, we evaluated each predictor individually (each being coded as centered variable). As suggested by the main analyses above, higher magnitudes were indeed associated with a higher preference for right responses (fixed effect estimate $=0.055 /$ number, $\mathrm{SE}=0.032), z=1.70, p=0.045, p_{p b}=0.036 .^{2}$ Surprisingly, even numbers were more likely to be dealt to the left side as compared to odd numbers (fixed effect estimate $=0.281, \mathrm{SE}=0.147$ ), indicating a reliably reversed MARC effect, $z=-1.91, p=0.028$, $p_{p b}=0.030$.

For model comparisons, we fitted a null model including only an intercept on the first level, an additive model with value and parity as independent predictors, and a saturated model with main effects as well as the two-way interaction. In a first step, we compared the null model to the additive model. This comparison yielded a marginally significant effect in favor of the additive model $\chi^{2}(2)=5.42, p=0.067, p_{p b}=0.069$, indicating that the two additional parameters did indeed add explanatory value. Further including the interaction effect, however, did not improve model fit significantly, $\chi^{2}(1)=0.01, p=0.941$, $p_{p b}=0.929$.

\section{DISCUSSION}

We investigated the effects of different characteristics of numbers (values of playing cards) on biases in fair distribution behavior. Indeed, we found evidence for such systematic biases in a free

${ }^{2}$ Based on the comments of a reviewer, the model comparison was repeated using parametric bootstrapping with 1000 simulations, using the PBmodcomp() function of the R package pbkrtest (Halekoh and Højsgaard, 2014). choice experiment: Participants read out loud a rummy card's value and announced their spatial assignment to a leftward or rightward positioned player. Without applying explicit strategies, participants failed to distribute cards in a statistically fair way and assigned a mean benefit of two cards or 16 points to the right player. In line with recent findings from the linguistic markedness and spatial-numerical associations of response codes effects, we hypothesized such a pattern to be partly driven by odd and high numbers being associated with rightward oriented action codes.

In the following exploratory analyses, we aimed at dismantling SNARC and MARC-like effects on response decisions at an individual, trial-wise level. Indeed, we found some evidence for the regular SNARC effect, but the data also indicated a reversed MARC effect with odd numbers being more likely to be distributed to the right player and even numbers being more likely to be distributed to the left player. Although this latter finding certainly comes unexpected, several recent studies cast doubt on a stable left-right association of odd and even numbers. Rather, the direction of the MARC effect seems to depend on task rules, i.e., affirmative answers seem to be generally compatible with right response codes and might override the parity-driven code of an odd number (Cho and Proctor, 2007). Further, Nuerk et al. (2005) observed the MARC effect to be altered by stimulus and experimental settings: Whereas participants showed a usual MARC effect for number words when the experiment started with Arabic notation digits, this effect was reversed when the experiment started with dice-dot patterns. In light of the apparent similarity of dice patterns and the patterns printed on the playing cards of the current experiment (see Figure 1), one might speculate that such gambling-related stimuli might generally elicit a reversed linguistic markedness of parity; however, Chang and Gibson (2011) found a regular odd-even effect in Sudoku puzzles and future studies are needed to clarify these speculations and investigate the underlying mechanisms.

Such flexibility of the MARC effect further seems likely in light of various findings on flexible coding of the related SNARC effect. For instance, the SNARC effect is influenced by interindividual characteristics such as finger counting habits (Fischer, 2008), cultural aspects such as reading direction (Shaki et al., 2009; Domahs et al., 2010) as well as sex (Bull et al., 2013) and age (Wood et al., 2008). The MARC effect, similarly, was recently found reversed for left-handers (Huber et al., 2014), which supports a body-specificity account (Casasanto, 2009) rather than a linguistic markedness account (Nuerk et al., 2004). Furthermore, the SNARC effect is also modulated by short-term, contextual factors such as recently encountered episodes (sequence effects: Pfister et al., 2013), number usage (number placement in text: Fischer et al., 2010; on a ruler vs. clock face: Bächtold et al., 1998) and current number range (Dehaene et al., 1993; Fias et al., 1996). 


\section{GENERAL PLACEMENT PREFERENCES}

Of course, the overall preference for the right card slot of our mostly right-handed participants also reminds of robust phenomena unrelated to the processing of numerical stimuli such as turning biases when confronted with a decision to take either a left or a right turn (Liederman and Kinsbourne, 1980; Güntürkün, 2003; cf. Shaki and Fischer, 2014, for the interplay of number processing and turning during walking). Furthermore, physical positioning was shown to produce more positive attitudes for rightward placed items (Nisbett and Wilson, 1977; Choi and Myer, 2012). Vice versa, positive abstract concepts were associated with right space for right-handed participants (Casasanto, 2009). In fact, a vast amount of marketing literature is concerned with devaluation of laterally placed items (Dittrich and Klauer, 2012), which is at times confounded with a desirable perception of magnitude (i.e., heaviness perception; Deng and Kahn, 2009) or automatic price and quality inferences (i.e., expensive and highquality items on the right end; Valenzuela and Raghubir, 2009). For free choice actions, goal keepers were found more likely to dive to the right during shoot-outs and under pressure (Roskes et al., 2011; but see Price and Wolfers, 2014), which was taken to document approach motivation (Roskes et al., 2014).

\section{HANDEDNESS-DEPENDENT PLACEMENT PREFERENCES}

Already for spontaneous turning biases, stronger right-sided head-turning was documented for right-handed than for lefthanded participants (Ocklenburg and Güntürkün, 2009). Similarly, positive abstract concepts were associated with rightward space for right-handers, but left-handed participants with similar linguistic experience (i.e., use of metaphors) showed a reversed association of abstract concepts and space (Casasanto, 2009), suggesting that bodily experiences might shape valence-specific placement preferences. In a large Moroccan sample that exhibited strong taboos against the use of left hands, the implicit spacevalence association was found effectively identical compared to a Spain sample (de la Fuente et al., 2014), but explicit measures (i.e., good-is-right rating and ratio of right/left-handers) were larger in the Arab population. Thus, handedness and according interactions with the external world appear to be valid candidates in explaining general and explicit spatial mappings of valence.

Given the data at hand, we cannot provide evidence for culture or hand-experience specific modulations. However, valence-space and value-space associations are not necessarily interchangeable, despite a possible positive connotation of playing cards or numbers in general. For mere numbers, reversing the polarity of a response side through response eccentricity did not affect spatial-numerical associations (Santiago and Lakens, 2014), suggesting that the link between numbers and space is not (exclusively) driven by their value-valence correspondence (i.e., polarity correspondence; Proctor and Cho, 2006). Another study even suggested magnitude to underlie spatial valence representations (Holmes and Lourenco, 2011). Furthermore, number-space associations are manifold regarding the number's features (see Patro et al., 2014, for a recent taxonomy proposal at an early age), and we next discuss the possible interpretation of SNARC and MARC effects in terms of linguistic markedness.

\section{LINGUISTIC MARKEDNESS IN NUMBER PROCESSING?}

It is widely accepted that number processing includes a verbal component, as suggested by the triple-code model (Dehaene et al., 1993; Klein et al., 2014). Semantic features of the number (parity and magnitude) are activated automatically and can deteriorate unrelated task processing already in children of 10 years of age (Berch et al., 1999). As such, linguistic markedness of a verbal number-code, i.e., in form of the non-marked even parity feature, might facilitate equally non-marked responses, i.e., right actions (Nuerk et al., 2004). Arguably, in this experiment, the number of cards dealt to a player can be regarded an unspecific placement preference and explained a substantial proportion, but not all variance of differences in scores. Rather, the results from our exploratory analysis suggest that space-number associations further biased the distribution outcome, and that reversed spaceparity associations supported but space-magnitude associations counteracted the fair distribution.

For linguistic influences in the SNARC effect, instead of assuming an oriented mental number line (i.e., Göbel et al., 2001), it is similarly possible that magnitude is coded by opposed small/large polar or linguistic representations (c.f. Nuerk et al., 2004; Proctor and Cho, 2006). Facilitated left/right responses can be accounted for by corresponding pairs of markedness: The adjectives large and small are lexical opposites with large as the non-marked adjective (Jakobson, 1931; see also: Lehrer, 2008). Similarly, the adjective right is linguistically non-marked (Zimmer, 1964), and the correspondence of both non-marked (i.e., large and right) and marked (i.e., small and left) pairs would lead to the SNARC effect. Homogenous marked and nonmarked pairs should be responded to faster and they should more often be selected in a free choice paradigm. Consequently, with a decreasing marked property of small, the marked left response side was chosen less frequently. However, it is not clear how linguistic markedness can account for flexible magnitudespace and reversed parity-space associations; instead, a flexible, body-specific conceptual layer, i.e., in form of polarity or space, seems more likely. Obviously, participants were more cautious in distributing high-value (i.e., royal) cards more equally in order to distribute the cards fairly; nevertheless, magnitude-response correspondence, as indexed by the regular SNARC effect, could have effectively led to the observed right-bias.

Crucially, the interpretation of the SNARC effect in terms of polarity correspondence (Proctor and Cho, 2006) or verbal codes (Gevers et al., 2010) does not exclude the possibility of a visuo-spatial representation of magnitude. In line with the dual-coding framework of Paivio (1986), non-verbal and verbal representations can be processed referentially and activate each other. The observed SNARC effect in verbal and following motor responses can be attributed to such a referential activation. Possibly, a visuo-spatial representation was pronounced because our participants performed actual hand movements in a well-defined space, namely over a card-playing table.

We excluded two-digit and royal card stimuli from the mixedeffects SNARC and MARC models as too little is known about these indirectly magnitude-related stimuli at this time: Do they extend the mental number line similar to 0 (Pinhas and Tzelgov, 2012)? How are nominal two-digit numbers processed when part 
of this specific number range (Dehaene et al., 1993; Nuerk and Willmes, 2005; Nuerk et al., 2014) and does the pictorial presentation, i.e., of a king vs. a jack, trigger marked representations other than the rule-based card value?

Notwithstanding these open issues, a range of recent papers addressed the linkages of brain mechanisms devoted to language and action, respectively, and elaborated these linkages in several frameworks to accommodate for SNARC and MARC effects (e.g., Pulvermüller, 2005; Barsalou, 2008; Fischer, 2012). In case of the SNARC effect, interestingly, language or number processing is most likely only indirectly associated with motor system activations through magnitude processing (Fias et al., 1996) and magnitude-related spatial codes (Gevers et al., 2006) or verbal codes (Gevers et al., 2010). Still, this indirect loop was demonstrated sufficient to modulate deliberate action selection (Daar and Pratt, 2008; Ruiz Fernández et al., 2011). In this experiment, we further show that this bias even transfers to a more natural card playing scenario and is able to interfere with a fair distribution task.

\section{FAIR DECISIONS IN CARD DISTRIBUTION}

Although statistically the goal of fair distribution was not met, participants were mostly confident about their choices during debriefing and reported to have achieved the goal by deciding upon a subjective feeling of just distribution. This finding is in line with results on the egocentric fairness bias (Tanaka, 1999), stating that especially just world believers (Rubin and Peplau, 1975) consider their own behavior as fairer than other people's behavior. In relation to these findings, the perception of fairness might be considered biased by social demands (Blair, 2002), whereas actual fair behavior was counter-acted here by automaticity, i.e., number-space associations.

Several alternative explanations might also account for the observed general preference for the right player. In this regard, some limitations of the study have to be considered: Both the table coloring and the player characters were not counterbalanced and could have implied unidentified response tendencies ${ }^{3}$. The study sample was rather diverse regarding participants' age, sex, and handedness, which likely increased the variance of number-space associations. Future studies should more closely examine these characteristics' interactions with number-driven action decisions. By including the rummy card set, the stimuli used were, on one hand, of high external validity and allowed for instructing and investigating fair distribution behavior. On the other hand, the stimulus set by nature included two-digit and pictorial cards and thereby differs from previous studies. Nevertheless, we focused on single digits only in the mixed effects models analysis and thereby, the results of this analysis must be regarded exploratory and might underestimate the SNARC effect for the entire number range.

A closer look at single digits in the exploratory analysis pointed towards regular magnitude-space associations, but reversed parity-space associations. As such, automatic number magnitude processing emphasized a possible pre-existing preference bias by suggesting rightward (leftward) choices for high

${ }^{3}$ For effects of color on cognition, see Elliot and Maier (2014). We thank a reviewer for drawing our attention to this point. (low) value cards, resulting in higher scores. Given the full standard rummy card set, a regular MARC effect would have further emphasized responses favoring the right player. Placement preferences were increasingly identified in the literature, and the same is true for number-space associations. In a natural setting, it is likely that both types of bias affect choices, and our analysis confirms this view by the combination of identity-unspecific results (number of cards) and number specific results (scores and single-digit decision outcomes).

In conclusion, the results of our study support current views of actions as being influenced by language processing. During card distribution and while aiming at a fair and equal distribution, the participants' choices were still affected by linguistic or conceptual features of actual rummy cards, namely digit parity and magnitude. A regular SNARC and a reversed MARC effect emerged and ultimately supported the overall preference of a right player avatar. The successful transfer of these effects to a more natural setting emphasizes the importance of further understanding the (neural) mechanisms behind indirectly and directly actionrelated linguistic and conceptual influences on number processing. Understanding these mechanisms will allow for identifying in which situations number associations can systematically bias behavior and, consequently, a better understanding will allow for countering these biases.

\section{AUTHOR CONTRIBUTIONS}

PS and RP designed research; PS performed research; PS and RP analyzed data and wrote the paper.

\section{ACKNOWLEDGMENTS}

We acknowledge support by Deutsche Forschungsgemeinschaft and Open Access Publishing Fund of Tübingen University. We are grateful to Julia Schönrock for casting and recruiting suitable Playmobil ${ }^{\circledR}$ characters for the study.

\section{REFERENCES}

Bächtold, D., Baumüller, M., and Brugger, P. (1998). Stimulus-response compatibility in representational space. Neuropsychologia 36, 731-735. doi: 10.1016/S00283932(98)00002-5

Barsalou, L. (1999). Perceptions of perceptual symbols. Behav. Brain Sci. 22, 637660. doi: 10.1017/S0140525X99532147

Barsalou, L. W. (2008). Grounded cognition. Annu. Rev. Psychol. 59, 617-645. doi: 10.1146/annurev.psych.59.103006.093639

Bates, D., Maechler, M., Bolker, B., and Walker, S. (2014). Linear Mixed-Effects Models Using Eigen and S4. R Package version 1.1-7. Available at: http//CRAN.Rproject.org/package $=$ lme 4

Berch, D. B., Foley, E. J., Hill, R. J., and Ryan, P. M. (1999). Extracting parity and magnitude from Arabic numerals: developmental changes in number processing and mental representation. J. Exp. Child Psychol. 74, 286-308. doi: 10.1006/jecp.1999.2518

Blair, I. V. (2002). The malleability of automatic stereotypes and prejudice. Personal. Soc. Psychol. Rev. 6, 242-261. doi: 10.1207/S15327957PSPR0603_8

Bull, R., Cleland, A. A., and Mitchell, T. (2013). Sex differences in the spatial representation of number. J. Exp. Psychol. Gen. 142, 181-192. doi: 10.1037/a0028387

Casasanto, D. (2009). Embodiment of abstract concepts: good and bad in rightand left-handers. J. Exp. Psychol. Gen. 138, 351-367. doi: 10.1037/a0015854

Chang, H.-S., and Gibson, J. M. (2011). The odd-even effect in Sudoku puzzles: effects of working memory, aging, and experience. Am. J. Psychol. 124, 313-324. doi: 10.5406/amerjpsyc. 124.3.0313

Cho, Y. S., and Proctor, R. W. (2007). When is an odd number not odd? Influence of task rule on the MARC effect for numeric classification. J. Exp. Psychol. Learn. Mem. Cogn. 33, 832-842. doi: 10.1037/0278-7393.33.5.832 
Choi, J., and Myer, D. W. (2012). The effect of product positioning in a comparison table on consumers' evaluation of a sponsor. Mark. Lett. 23, 367-380. doi: $10.1007 / \mathrm{s} 11002-012-9162-9$

Daar, M., and Pratt, J. (2008). Digits affect actions: the SNARC effect and response selection. Cortex 44, 400-405. doi: 10.1016/j.cortex.2007.12.003

Dehaene, S., Bossini, S., and Giraux, P. (1993). The mental representation of parity and number magnitude. J. Exp. Psychol. Gen. 122, 371-396. doi: 10.1037/00963445.122.3.371

de la Fuente, J., Casasanto, D., Román, A., and Santiago, J. (2014). Can culture influence body-specific associations between space and valence? Cogn. Sci. doi: 10.1111/cogs.12177 [Epub ahead of print].

Deng, X., and Kahn, B. (2009). Is your product on the right side? The "location effect" on perceived product heaviness and package evaluation. J. Mark. Res. 46, 1-48. doi: 10.1509/jmkr.46.6.725

Dittrich, K., and Klauer, K. C. (2012). Does ignoring lead to worse evaluations? A new explanation of the stimulus devaluation effect. Cogn. Emot. 26, 193-208. doi: 10.1080/02699931.2011.570313

Domahs, F., Moeller, K., Huber, S., Willmes, K., and Nuerk, H.-C. (2010). Embodied numerosity: implicit hand-based representations influence symbolic number processing across cultures. Cognition 116, 251-266. doi: 10.1016/j.cognition. 2010.05.007

Eerland, A., Guadalupe, T. M., and Zwaan, R. A. (2011). Leaning to the left makes the Eiffel Tower seem smaller: posture-modulated estimation. Psychol. Sci. 22, 1511-1514. doi: 10.1177/0956797611420731

Elliot, A. J., and Maier, M. A. (2014). Color psychology: effects of perceiving color on psychological functioning in humans. Annu. Rev. Psychol. 65, 95-120. doi: 10.1146/annurev-psych-010213-115035

Fias, W., Brysbaert, M., Geypens, F., and D’Ydewalle, G. (1996). The importance of magnitude information in numerical processing: evidence from the SNARC effect. Math. Cogn. 2, 95-110. doi: 10.1080/135467996387552

Fischer, M. H. (2012). A hierarchical view of grounded, embodied, and situated numerical cognition. Cogn. Process. 13(Suppl. 1), S161-S164. doi: 10.1007/ s10339-012-0477-5

Fischer, M. H. (2008). Finger counting habits modulate spatial-numerical associations. Cortex 44, 386-392. doi: 10.1016/j.cortex.2007.08.004

Fischer, M. H. (2006). The future for SNARC could be stark. ... Cortex 42, 1066 1068. doi: 10.1016/S0010-9452(08)70218-1

Fischer, M. H., Mills, R. A., and Shaki, S. (2010). How to cook a SNARC: number placement in text rapidly changes spatial-numerical associations. Brain Cogn. 72, 333-336. doi: 10.1016/j.bandc.2009.10.010

Furnham, A., and Boo, H. C. (2011). A literature review of the anchoring effect. J. Socio Econ. 40, 35-42. doi: 10.1016/j.socec.2010.10.008

Gevers, W., Ratinckx, E., De Baene, W., and Fias, W. (2006). Further evidence that the SNARC effect is processed along a dual-route architecture: evidence from the lateralized readiness potential. Exp. Psychol. 53, 58-68. doi: 10.1027/16183169.53.1.58

Gevers, W., Santens, S., Dhooge, E., Chen, Q., Van den Bossche, L., Fias, W., et al. (2010). Verbal-spatial and visuospatial coding of number-space interactions. J. Exp. Psychol. Gen. 139, 180-190. doi: 10.1037/a0017688

Göbel, S., Walsh, V., and Rushworth, M. F. (2001). The mental number line and the human angular gyrus. Neuroimage 14, 1278-1289. doi: 10.1006/nimg.2001.0927

Güntürkün, O. (2003). Human behaviour: adult persistence of head-turning asymmetry. Nature 421, 711. doi: 10.1038/421711a

Halekoh, U., and Højsgaard, S. (2014). A Kenward-Roger approximation and parametric bootstrap methods for tests in linear mixed models-the R package pbkrtest. J. Stat. Softw. 59, 1-30.

Holmes, K. J., and Lourenco, S. F. (2011). Common spatial organization of number and emotional expression: a mental magnitude line. Brain Cogn. 77, 315-323. doi: 10.1016/j.bandc.2011.07.002

Huber, S., Klein, E., Graf, M., Nuerk, H.-C., Moeller, K., and Willmes, K. (2014). Embodied markedness of parity? Examining handedness effects on parity judgments. Psychol. Res. doi: 10.1007/s00426-014-0626-9 [Epub ahead of print].

Jakobson, R. (1931). "The structure of the Russian verb," in Russian and Slavic Grammar Studies, eds L. Waugh and M. Halle (Berlin: Mouton), 1-14.

Keehner, M., and Fischer, M. H. (2012). Unusual bodies, uncommon behaviors: individual and group differences in embodied cognition in spatial tasks. Spat. Cogn. Comput. 12, 71-82. doi: 10.1080/13875868.2012.659303

Klein, E., Suchan, J., Moeller, K., Karnath, H.-O., Knops, A., Wood, G., et al. (2014). Considering structural connectivity in the triple code model of numerical cognition: differential connectivity for magnitude processing and arithmetic facts. Brain Struct. Funct. doi: 10.1007/s00429-014-0951-1 [Epub ahead of print].

Lehrer, A. (2008). Markedness and antonymy. J. Linguist. 21, 397. doi: 10.1017/ S002222670001032X

Liederman, J., and Kinsbourne, M. (1980). The mechanism of neonatal rightward turning bias: a sensory or motor asymmetry? Infant Behav. Dev. 3, 223-238. doi: 10.1016/S0163-6383(80)80028-2

Loetscher, T., Bockisch, C. J., Nicholls, M. E. R., and Brugger, P. (2010). Eye position predicts what number you have in mind. Curr. Biol. 20, R264-R265. doi: 10.1016/j.cub.2010.01.015

Loetscher, T., Schwarz, U., Schubiger, M., and Brugger, P. (2008). Head turns bias the brain's internal random generator. Curr. Biol. 18, R60-R62. doi: 10.1016/ j.cub.2007.11.015

Mussweiler, T., and Englich, B. (2003). Adapting to the Euro: evidence from bias reduction. J. Econ. Psychol. 24, 285-292. doi: 10.1016/S0167-4870(03)00015-1

Nisbett, R., and Wilson, T. (1977). Telling more than we can know: verbal reports on mental processes. Psychol. Rev. 84, 231-259. doi: 10.1037/0033-295X. 84.3.231

Northcraft, G. B., and Neale, M. A. (1987). Experts, amateurs, and real estate: an anchoring-and-adjustment perspective on property pricing decisions. Organ. Behav. Hum. Decis. Process. 39, 84-97. doi: 10.1016/0749-5978(87)90046-X

Nuerk, H.-C., Iversen, W., and Willmes, K. (2004). Notational modulation of the SNARC and the MARC (linguistic markedness of response codes) effect. Q. J. Exp. Psychol. A 57, 835-863. doi: 10.1080/02724980343000512

Nuerk, H.-C., Moeller, K., Klein, E., Willmes, K., and Fischer, M. H. (2011) Extending the mental number line: a review of multi-digit number processing. Z. Psychol. 219, 3-22. doi: 10.1027/2151-2604/a000041

Nuerk, H.-C., Moeller, K., and Willmes, K. (2014). "Multi-digit number processing," in Oxford Handbook of Mathematical Cognition, eds R. Cohen Kadosh and A. Dowker (Oxford: Oxford University Press), 1-20.

Nuerk, H.-C., and Willmes, K. (2005). On the magnitude representations of twodigit numbers. Psychol. Sci. 47, 52-72.

Nuerk, H.-C., Wood, G., and Willmes, K. (2005). The universal SNARC effect: the association between number magnitude and space is amodal. Exp. Psychol. 52, 187-194. doi: 10.1027/1618-3169.52.3.187

Ocklenburg, S., and Güntürkün, O. (2009). Head-turning asymmetries during kissing and their association with lateral preference. Laterality 14, 79-85. doi: 10.1080/13576500802243689

Paivio, A. (1986). Mental Representations: A Dual-Coding Approach. New York: Oxford University Press.

Patro, K., Nuerk, H.-C., Cress, U., and Haman, M. (2014). How number-space relationships are assessed before formal schooling: a taxonomy proposal. Front. Psychol. 5:419. doi: 10.3389/fpsyg.2014.00419

Pfister, R., and Janczyk, M. (2013). Confidence intervals for two sample means: calculation, interpretation, and a few simple rules. Adv. Cogn. Psychol. 9, 74-80.

Pfister, R., Schroeder, P. A., and Kunde, W. (2013). SNARC struggles: instant control over spatial-numerical associations. J. Exp. Psychol. Learn. Mem. Cogn. 39, 1953 1958. doi: $10.1037 / \mathrm{a} 0032991$

Pinhas, M., and Tzelgov, J. (2012). Expanding on the mental number line: zero is perceived as the "smallest." J. Exp. Psychol. Learn. Mem. Cogn. 38, 1187-1205. doi: $10.1037 / \mathrm{a} 0027390$

Porter, T. M. (1996). Trust in Numbers: The Pursuit of Objectivity in Science and Public Life. Princeton, NJ: Princeton University Press.

Price, J., and Wolfers, J. (2014). Right-oriented bias: a comment on roskes, sligte, shalvi, and de dreu (2011). Psychol. Sci. 25, 2109-2111. doi: 10.1177/ 0956797614536738

Proctor, R. W., and Cho, Y. S. (2006). Polarity correspondence: a general principle for performance of speeded binary classification tasks. Psychol. Bull. 132, 416442. doi: 10.1037/0033-2909.132.3.416

Pulvermüller, F. (2005). Brain mechanisms linking language and action. Nat. Rev Neurosci. 6, 576-582. doi: 10.1038/nrn1706

Roskes, M., Sligte, D., Shalvi, S., and De Dreu, C. K. W. (2014). Does approach motivation induce right-oriented bias? Reply to price and wolfers (2014) Psychol. Sci. 25, 2112-2115. doi: 10.1177/0956797614547919

Roskes, M., Sligte, D., Shalvi, S., and De Dreu, C. K. W. (2011). The right side? Under time pressure, approach motivation leads to right-oriented bias. Psychol. Sci. 22, 1403-1407. doi: 10.1177/0956797611418677 
Rubin, Z., and Peplau, L. A. (1975). Who Believes in a Just World? J. Soc. Issues 31, 65-89. doi: 10.1111/j.1540-4560.1975.tb00997.x

Ruiz Fernández, S., Rahona, J. J., Hervás, G., Vázquez, C., and Ulrich, R. (2011). Number magnitude determines gaze direction: spatial-numerical association in a free-choice task. Cortex 47, 617-620. doi: 10.1016/j.cortex.2010.10.006

Santiago, J., and Lakens, D. (2014). Can conceptual congruency effects between number, time, and space be accounted for by polarity correspondence? Acta Psychol. (Amst.) doi: 10.1016/j.actpsy.2014.09.016 [Epub ahead of print].

Shaki, S., and Fischer, M. H. (2014). Random walks on the mental number line. Exp. Brain Res. 232, 43-49. doi: 10.1007/s00221-013-3718-7

Shaki, S., Fischer, M. H., and Petrusic, W. M. (2009). Reading habits for both words and numbers contribute to the SNARC effect. Psychon. Bull. Rev. 16, 328-331. doi: 10.3758/PBR.16.2.328

Tanaka, K. (1999). Judgments of fairness by just world believers. J. Soc. Psychol. 139, 631-638. doi: 10.1080/00224549909598423

Tschentscher, N., Hauk, O., Fischer, M. H., and Pulvermüller, F. (2012). You can count on the motor cortex: finger counting habits modulate motor cortex activation evoked by numbers. Neuroimage 59, 3139-3148. doi: 10.1016/ j.neuroimage.2011.11.037

Tversky, A., and Kahneman, D. (1974). Judgment under uncertainty: heuristics and biases. Science 185, 1124-1131. doi: 10.1126/science.185.4157.1124

Valenzuela, A., and Raghubir, P. (2009). Are top-bottom inferences conscious and left-right inferences automatic? implications for shelf space positions. HEC Paris INSEAD Fontainebleau. 19, 185-196.
Wood, G., Willmes, K., Nuerk, H.-C., and Fischer, R. (2008). On the cognitive link between space and number: a meta-analysis of the SNARC effect. Psychol. Sci. Q. 50, 489-525.

Zimmer, K. E. (1964). Affixal negation in English and other languages: an investigation of restricted productivity. Word 20(2), Monograph No. 5 .

Conflict of Interest Statement: The authors declare that the research was conducted in the absence of any commercial or financial relationships that could be construed as a potential conflict of interest.

Received: 22 November 2014; accepted: 16 February 2015; published online: 20 March 2015.

Citation: Schroeder PA and Pfister $R$ (2015) Arbitrary numbers counter fair decisions: trails of markedness in card distribution. Front. Psychol. 6:240. doi: 10.3389/fpsyg.2015.00240

This article was submitted to Developmental Psychology, a section of the journal Frontiers in Psychology.

Copyright (c) 2015 Schroeder and Pfister. This is an open-access article distributed under the terms of the Creative Commons Attribution License (CC BY).

The use, distribution or reproduction in other forums is permitted, provided the original author(s) or licensor are credited and that the original publication in this journal is cited, in accordance with accepted academic practice. No use, distribution or reproduction is permitted which does not comply with these terms. 